\title{
Sparse Error Gait Image: A New Representation For Gait Recognition
}

\author{
Tanmay Tulsidas Verlekar \\ Instituto de Telecomunicações \\ Instituto Superior Técnico, \\ Universidade de Lisboa, \\ Lisboa, Portugal \\ Tanmay.Verlekar@lx.it.pt
}

\author{
Paulo Lobato Correia \\ Instituto de Telecomunicações \\ Instituto Superior Técnico, \\ Universidade de Lisboa, \\ Lisboa, Portugal \\ plc@1x.it.pt
}

\author{
Luís Ducla Soares \\ Instituto de Telecomunicações \\ Instituto Universitário de Lisboa \\ (ISCTE-IUL), \\ Lisboa, Portugal \\ lds@1x.it.pt
}

\begin{abstract}
The performance of a gait recognition system is very much related to the usage of efficient feature representation and recognition modules. The first extracts features from an input image sequence to represent a user's distinctive gait pattern. The recognition module then compares the features of a probe user with those registered in the gallery database. This paper presents a novel gait feature representation, called Sparse Error Gait Image (SEGI), derived from the application of Robust Principal Component Analysis (RPCA) to Gait Energy Images (GEI). GEIs obtained from the same user at different instants always present some differences. Applying RPCA results in lowrank and sparse error components, the former capturing the commonalities and encompassing the small differences between input GEIs, while the larger differences are captured by the sparse error component. The proposed SEGI representation exploits the latter for recognition purposes. This paper also proposes two simple approaches for the recognition module, to exploit the SEGI, based on the computation of a Euclidean norm or the Euclidean distance. Using these simple recognition methods and the proposed SEGI representation gait recognition, results equivalent to the state-of-the-art are obtained.
\end{abstract}

Keywords-Robust Principal Component Analysis; Gait Recognition; Biometrics.

\section{INTRODUCTION}

Gait is a biometric trait which is a defined as the way a person walks. While walking, a person repeats its walking gestures along the so-called gait cycle, which can be considered as a fundamental unit of gait. Features extracted from one or several complete gait cycles can be used to attempt user recognition. However, unlike more traditional biometric traits, such as fingerprint or iris, which are unique among large populations, the observable gait information may be more suitable for using with small populations, as discussed in [1]. In scenarios where the population size is significantly large, gait may nevertheless be used as a part of a multi-modal recognition system, to improve results and the efficiency of the recognition process. Also, in scenarios such as surveillance, where video sequences are acquired over a distance and where it is easy to hide eyes or faces, gait provides advantages over traditional biometric traits. Gait does not require active user

This work was partially supported by Instituto de Telecomunicações under Fundação para a Ciência e Tecnologia Grant UID/EEA/50008/2013 cooperation and can be used to perform recognition even when the faces or eyes are not visible [1].

The general architecture of a gait recognition system includes two main modules: i) Feature Representation and ii) Recognition. The feature representation module converts the available walking silhouette sequences, obtained through initial preprocessing, into a set of features capturing a user's distinctive gait. Recognition can then be performed by matching features obtained from a probe gait sequence against those previously stored in the database gallery. Thus, the performance of a recognition system can be improved by improving the quality of the features and/or by improving the recognition methods. The work presented in this paper mainly focuses on the feature representation module, notably by proposing a novel gait representation, called Sparse Error Gait Image (SEGI). Using SEGI it is possible to improve recognition results, when compared with the available gait representation alternatives.

In the literature, gait recognition methods can be broadly categorized into two groups: 1) model based and 2) appearance based methods.

Model based methods try to model the body or motion of a user based on some prior knowledge; examples include the work presented in [2], where a Kinect camera is used to obtain the 3D-skeleton of a user. Static features such as distances between joints and joint angles can be extracted from the 3Dskeleton and used for recognition. The performance of such a model is further improved by additionally using dynamic features such as speed, stride length and variation of barycenter for recognition [3]. Similar features can be computed using multiple cameras [4]. Another example uses a 3D model of a user to render a set of $2 \mathrm{D}$ projections, which are used for recognition [5]. In [6], a 3D model of a user, reconstructed from images obtained from multiple cameras, is used for recogniton. The methods belonging to this group are less sensitive to background noise, once the model has been created, and perform well under viewpoint changes. However, they are adversely affected by occlusions, as they rely on accurate detection of keypoints such as joints. The use of 3D models can also result in computationally expensive methods, 
and the use of multiple cameras may not be possible in some application scenarios.

Some other model based methods rely on features that are robust to viewpoint changes, such as the work presented in [7], which uses hip, knee and ankle positions obtained from a gait sequence for any view to model the angular measurements of a user in lateral view. Similarly, the work presented in [8] models the head and feet position trajectories of a user, over a gait cycle, in a lateral view. These lateral view projections obtained from a random view are used for recognition. However, these methods rely on anatomy ratios to identify knee and feet positions, which are usually approximated. Thus they do not operate well under occlusion or large changes in view direction.

The second group of gait recognition methods includes the appearance based methods that rely on spatiotemporal features obtained from observed gait sequences without the use of any prior knowledge. A popular feature used in such methods is called Gait Energy Image (GEI) which is proposed in [9]. It is obtained by averaging cropped binary silhouettes over a gait cycle. It is used in [10] to perform recognition along different views using Euclidean distance. The performance is further improved in [11] by setting weights to different regions of the GEI. The GEI is also used in [12] to generate a view transformation model (VTM) which learns the mapping between different views to perform recognition. The method is improved by using it along with a support vector machine in [13] and a multi-layer perceptron in [14]. The performance of the VTM is also improved by using different gait features such as Radon transform-based energy images in [15] and a view fusion feature in [16]. However, VTM methods cannot determine the view angle apriori. View detection is performed as an initial step in [17] by computing entropy over the leg region of a probe GEI and matching it against the gallery. This is followed by user recognition performed using Random Subspace Learning. The work is improved in [18] by applying a Gaussian filter to the GEIs at different scales before using them as a feature for recognition. The work presented in [19] performs view detection using a Gaussian process (GP) classifier on a GEI, followed by canonical correlation analysis (CCA) for recognition. On the other hand, [20] analyzes the contour of a gait texture image for view detection. It then computes dissimilarity vectors using a prototype over the GEIs, which are used as features for recognition. A different approach groups GEIs from multiple views to learn a subspace that represents the set, thus avoiding the need for view tagging. An example of this approach is presented in [21], which frames the subspace in a Grassmann manifold.

Other popular features used in appearance based methods include the Gait Entropy Image presented in [22], [23], which is generated by computing the entropy over a GEI, as well as the Poisson Random Walk GEI presented in [24], which is computed by applying a Poison Random Walk to binary silhouettes. The work presented in [25] summarizes all the gait representations available in the literature and tests their performance using a simple k-nearest-neighbour approach. The results show room for improvement in the feature representation module of a gait recognition system.
This paper presents a new representation for gait recognition, denoted as Sparse Error Gate Image (SEGI). It is derived by applying Robust Principal Component Analysis (RPCA) [26] to the well-known gait energy image (GEI). To our knowledge, RPCA has not been previously applied for gait recogniton, although it has been successfullly applied in face recogniton applications [27][28], where the sparse error generated by RPCA is used to extract texture information to be used as a feature for face recognition. Although gait cannot rely on texture features, as these would be mostly related to clothing, which changes along time, the sparse error can still provide some useful information, as captured by the proposed SEGI representation.

In this paper, two different approaches are presented to use the proposed SEGI representation for gait recognition. The first generates a set of probe SEGIs for a given probe GEI, with respect to each user in the gallery database, and recognition is performed by looking for the minimum sparse error Euclidean norm. The second approach compares the Euclidean distance between probe and gallery SEGIs. Using the second approach provides results equivalent to state-of-the-art gait recognition methods. The simplicity of the recognition approaches highlights the effectiveness of the proposed SEGI representation, and even better performance can be expected by using more advanced recognition methods.

The remainder of the paper is organized as follows. Details of RPCA are discussed in section II. The proposed methods are presented in section III, with the corresponding experimental results being reported in section IV. Finally, section V provides some conclusions and directions for future work.

\section{RoBUSt PRINCIPAL COMPONENT ANALYSIS (RPCA)}

Consider a data matrix $D \in R^{m \times n}$ corrupted by sparse error $E$, i.e., an error matrix whose entries are mostly zero, but the non-zero entries are practically unbounded. The data matrix $D$ can be represented as: $D=L+E$, where $L$ is the underlying lowrank matrix. RPCA, proposed in [26], decomposes the data matrix $D$ into one low-rank matrix $L$ and one sparse error matrix $E$. This decomposition can be defined as:

$$
\min _{L, E} \operatorname{rank}(L)+\gamma\|E\|_{o} \text { subject to } D=L+E
$$

where $\|.\|_{o}$ is the counting norm (i.e., the number of non-zero entries in the matrix) and $\operatorname{rank}(L)$ is the number of linearly independent rows (or columns) in the matrix $L . \gamma$ is a regularization parameter.

However, the minimization of rank is a nonconvex problem. Thus, a convex relaxation of (1) is proposed in [26], which can be defined as:

$$
\min _{L, E}\|L\|_{*}+\lambda\|E\|_{1} \quad \text { subject to } \quad D=L+E
$$

where $\|L\|_{*}=\sum_{i} \sigma_{i}(L)$ denotes the nuclear norm of the matrix $L$, i.e., the sum of the singular values of $L$, and $\|E\|_{l}=\sum_{i j}\left|E_{i j}\right|$ denotes the $l_{1}$-norm of $E . \lambda$ is a regularization parameter, which is always set to $1 / n$, where $n=\left(\max \left(n_{1}, n_{2}\right)\right)^{1 / 2}$ and $n_{1}, n_{2}$ are the dimensions of $L$. 
The optimization problem can then be solved using the method of augmented Lagrange multipliers (ALM), making it possible to obtain the sparse component $E$, as long as the rank of $L$ is not too large. However, the ALM method has a high computational complexity due to the singular value decomposition performed during optimization. Thus, an alternative method, proposed in [29], can be used, which uses a simple alternating minimization algorithm for solving a minor variation of the original RPCA problem. This significantly reduces the computational complexity, while maintaining the quality of the decomposition results. It is used in this paper to obtain the SEGI representation.

\section{PROPOSED REPRESENTATION}

The new gait representation, SEGI, is presented in this section. The steps to follow to compute the SEGI are explained. Its advantages over the popular GEI gait representation are discussed. Then, two simple ways to use the proposed SEGI to perform gait recognition are presented.

\section{A. Sparse Error Gait Image (SEGI)}

To compute the proposed SEGI representation, RPCA is applied to a set of GEIs. Each gait energy image (GEI) [13] is obtained from a gait video sequence by averaging the person's gait silhouettes over an integer number of gait cycles $N$.

When RPCA is applied to a set of GEIs, it computes two types of outputs, a low rank component and a sparse error component, for each of the input GEIs. The low rank component is generated by projecting the GEIs onto the principal components identified by RPCA. The number of principal components is controlled by the constraints of the optimization problem in (2). Thus, minor variations in the GEI are incorporated into the low rank component. Since the low rank component does not contain any distinctive features that can be used to perform gait recognition, it can be ignored for the purposes of this paper. The sparse error component captures the larger variations in the GEIs, whose representation in the RPCA space are far from the low rank component. The sparse error component therefore provides a representation of the difference between a given GEI and the rest of the available GEIs, as represented by the low rank component. The sparse error corresponding to a given input GEI is the proposed SEGI representation. An illustration of a SEGI is included in the top of Fig. 1. Unlike a GEI, which can be computed using silhouettes of a single gait sequence, SEGI is generated by using at least 2 GEIs.
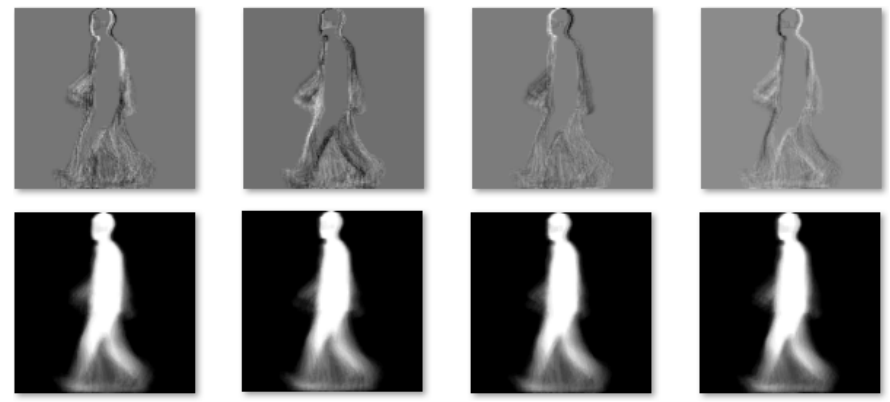

Fig. 1. SEGI (top) and the corresponding GEI (bottom).

\section{B. Galery and Probe Sparse Error Gait Images Computation}

To successfully use the SEGI representation for recognition, the architecture presented in Fig. 2 can be followed. The GEIs of each registered user are grouped and RPCA is applied to them to build the gallery SEGIs of each user. This is repeated for all the users.

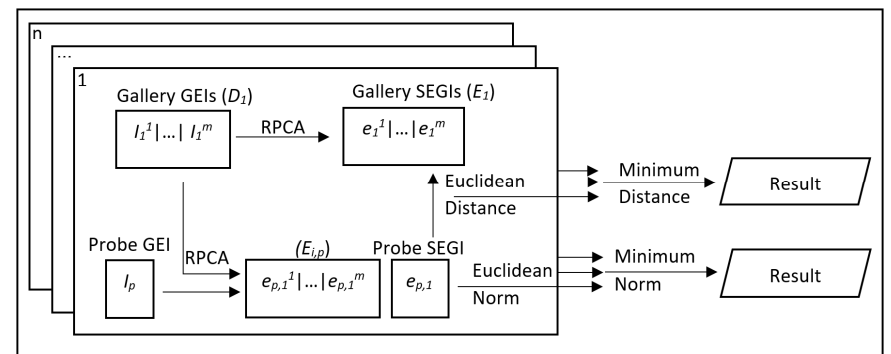

Fig. 2. System architecture.

Consider a database with $n$ users; for each user, $m$ gait sequences, represented as GEIs, are registered in the database. Each GEI is represented in the form of a 1D column vector by concatenating the rows one after another and then transposing the result. A matrix $D_{i}$ can be constructed for each user $i$ by appending together the vectorised gallery GEIs $I^{1, \ldots, m}$ belonging to the same user, according to (3):

$$
D_{i}=\left[\operatorname{vec}\left(I_{i}{ }^{l}\right)|\ldots| \operatorname{vec}\left(I_{i}^{m}\right)\right]
$$

To obtain the sparse error gait images corresponding to each of the gallery GEIs, for each user $i$, the sparse error matrix $E_{i}$ is computed by applying RPCA to matrix $D_{i}$. The resulting matrix $E_{i}$ contains the vectorised gallery SEGI, $e_{l}^{j}$, of the corresponding gallery GEI $I_{i}^{j}, j \in 1, \ldots, m$, according to (4):

$$
E_{i}=\left[\operatorname{vec}\left(e_{i}^{l}\right)|\ldots| \operatorname{vec}\left(e_{i}^{m}\right)\right]
$$

The vectorised gallery SEGIs, $E_{i}$, thus obtained are stored in the gallery database along with the matrix $D_{i}$, for all users $i$.

Since gallery SEGIs result from applying RPCA to a group of gallery GEIs belonging to the same user, these variations are expected to be small and similar for the same user. An example is shown in Fig. 3, where the intensity of each pixel is displayed following a column-wise raster scan and the middle gray level (128) in the left figure corresponds to a zero intensity level in the plot.
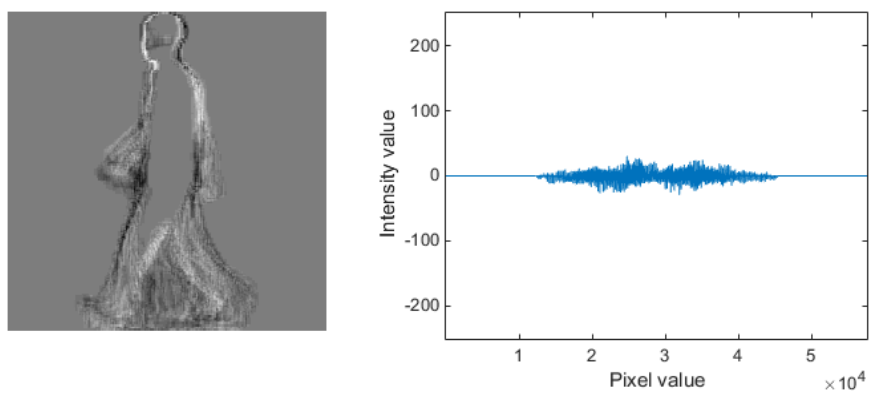

Fig. 3. SEGI representation and the corresponding intensity values for a gallery GEI. 
To perform recognition for a probe GEI, its SEGI representation is needed. Consider a probe GEI $I_{p}$, represented as a $1 \mathrm{D}$ column vector. To compute the probe SEGI, a context is required, and it will be the set of GEIs of the gallery user that is being considered as a candidate for matching. Therefore, the computation of the probe SEGI involves appending the vectorised probe GEI, $I_{p}$, to the considered user's matrix $D_{i}$ according to (5):

$$
D_{i, p}=\left[\operatorname{vec}\left(I_{i}{ }^{l}\right)|\ldots| \operatorname{vec}\left(I_{i}^{m}\right) \mid \operatorname{vec}\left(I_{p}\right)\right]
$$

RPCA can then be applied to each matrix $D_{i, p}$ to obtain the corresponding sparse error matrix $E_{i, p}$. The probe SEGI, representing the dissimilarity between the probe $p$ and the gallery user $i$ is represented by $e_{p, i}$, according to (6):

$$
E_{i, p}=\left[\operatorname{vec}\left(e_{i}{ }^{l}\right)|\ldots| \operatorname{vec}\left(e_{i}{ }^{m}\right) \mid \operatorname{vec}\left(e_{p, i}\right)\right]
$$

After applying RPCA to every matrix $D_{i, p}$, probe SEGIs with respect to each user registered in the gallery database are obtained, as $e_{p, i}$. When the probe SEGI is generated with respect to a genuine user the variation is small and similar to the gallery SEGIs, as illustrated in Fig. 4. However, when the probe SEGI is generated with respect to an impostor user, then the SEGI differences are significantly larger, as illustrated in Fig. 5. Apart from these variations, the appearance of the SEGI is also altered. Thus, to identify a user, the probe SEGI can be used directly or, as an alternative, the change in appearance with respect to the gallery SEGIs can also be considered. These are the two approaches that will be proposed next.
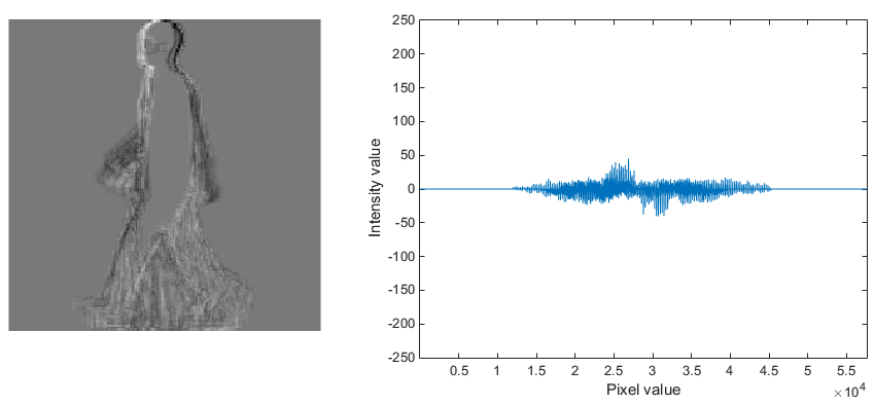

Fig. 4. Probe SEGI representation and the corresponding intensity values obtained with respect to a genuine user.
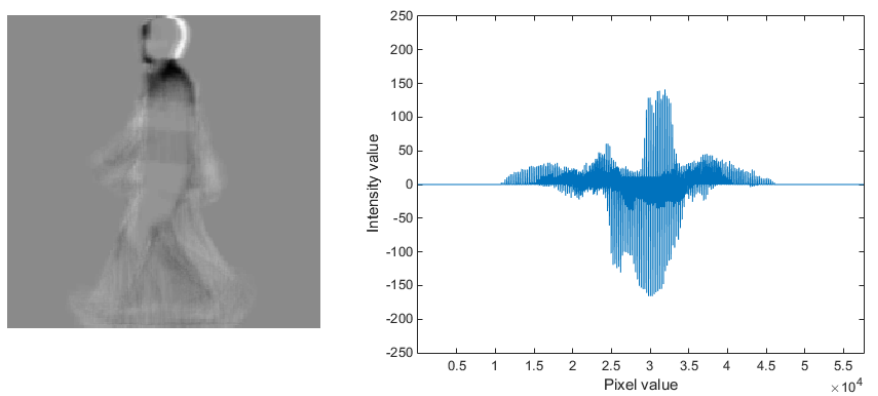

Fig. 5. Probe SEGI representation and the corresponding intensity values obtained with respect to an impostor user.

\section{Using Sparse Error Gait Image for Gait Recognition}

To perform recognition with a probe GEI, this can be done: i) by computing the Euclidean norm of the probe SEGIs obtained with respect to each user, or ii) by computing the Euclidean distances between the probe and the gallery SEGIs.

\section{Euclidean Norm Approach}

In this approach, the probe SEGI is generated using the gallery GEIs according to (5) and (6). Next, the Euclidean norm of the probe SEGI with respect to each user is computed, as represented in Fig. 2. The probe user is recognized as the user for which the computed probe SEGI has the smallest Euclidean norm, according to (7):

$$
\text { User= } \arg \min _{i}\left\|e_{p, i}\right\|^{2}
$$

This approach performs well because the SEGI represents how different a given probe GEI is from the gallery GEIs with respect to each user. For a genuine user, the probe GEI will not differ significantly from its representatives in the gallery and thus will lead to a small sparse error. On the other hand, a probe GEI from an impostor, will lead to a significantly large sparse error. To evaluate how large the sparse error is, its Euclidean norm can be computed and recognition can be based on it.

\section{Euclidean Distance Approach}

Similarly to the previous approach, the probe SEGI is generated using the gallery GEIs, according to (5) and (6). Next, the Euclidean distance between the probe and the gallery SEGIs is computed with respect to each user, as in (8):

$$
\operatorname{Dist}_{i, j}=\left\|e_{i}^{j}-e_{p, i}\right\|^{2} \quad j \in 1, \ldots, m
$$

Recognition is performed by selecting the user having the smallest SEGI Euclidean distance, as in (9):

$$
\text { User }=\arg \min _{i}\left(\text { Dist }_{i, j}\right)
$$

When the probe SEGI is from the correct user, its principal components will be aligned with those of the gallery SEGIs. Their low rank components will be similar and thus the appearance of the probe SEGI will be similar to the gallery SEGIs. For an impostor user, this will not be the case, as the alignment of the probe principal components will be different from those of the gallery, resulting in significantly different SEGIs.

\section{RESULTS}

To test the performance of the proposed approaches, the dataset B of CASIA gait database, collected by the Institute of Automation of the Chinese Academy of Sciences [30] is used. The database consists of 124 users walking in 11 different directions, with a difference of $18^{\circ}$ between them. Each user has 10 associated sequences. Among them, 6 sequences are considered normal, in the sense that the appearance of the user does not change significantly with respect to normal walking conditions, while the remaining 4 correspond to sequences where the appearance of the user has changed significantly. 
As the work in this paper is concerned with presenting the effectiveness of the proposed SEGI gait representation, at this stage the use of the database is limited to lateral $\left(90^{\circ}\right)$ sequences, without alterations to the appearance. The problems of change in appearance and view invariance will be addressed in future work. Therefore, only the 6 normal walking sequences are used. For the first test, following the setup used in the literature, the first 4 normal sequences are used for training and the remaining 2 normal sequences are used for testing. The corresponding results, along with results from state-of-the-art methods, are presented in TABLE I.

TABLE I. CORRECT CLASSIFICATION RATE OF THE STATE-OF-THE-ART METHODS

\begin{tabular}{|l|c|}
\hline \multicolumn{1}{|c|}{ Methods } & $\begin{array}{c}\text { Correct recognition rate } \\
(\%)\end{array}$ \\
\hline Dissimilarity Vector+KNN [20] & 80.2 \\
\hline CGI [25] & 87.0 \\
\hline GEI [25] & 90.0 \\
\hline GEnI [22] & 92.3 \\
\hline GPPE [23] & 93.3 \\
\hline FDEI [25] & 94.0 \\
\hline AMI+Weight Integration [11] & 97.0 \\
\hline Proposed SEGI (Euclidean Norm) & 98.4 \\
\hline Proposed SEGI (Eucliden Distance) & 99.2 \\
\hline Multiscale GEI+WRSL [18] & 100.0 \\
\hline $\begin{array}{l}\text { Proposed SEGI (Eucliden Distance, } \\
\text { Using 5 Training Sequences) }\end{array}$ & 100.0 \\
\hline
\end{tabular}

In the table, the second column reports performance results. The performance of the proposed methods is equivalent to the state-of-the-art, with the best performance being achieved by the multiscale method [18], which uses a complex random subspace learning method to perform recognition. It should be noted that the proposed method considers a very simple recognition method, combined with the proposed SEGI representation. The performance of the method can thus be attributed to the effectiveness of SEGI for representing a user's gait.

A second test is conducted to analyze the performance of the proposed approaches based on the number of available gallery sequences for each user. The test is conducted by starting with a single gallery sequence per user. The number of gallery sequences per user is increased, from 1 to a maximum of 5 , since 6 normal walking sequences are available in dataset B of CASIA gait database. Results are included in Fig. 6 as a plot of correct recognition rate versus number of gallery sequences per user. It can be seen that using the Euclidean distance provides significantly better results than the even simpler Euclidean norm approach. If sufficient SEGI representations are made available in the gallery, a correct recognition rate of $100 \%$ can be obtained.

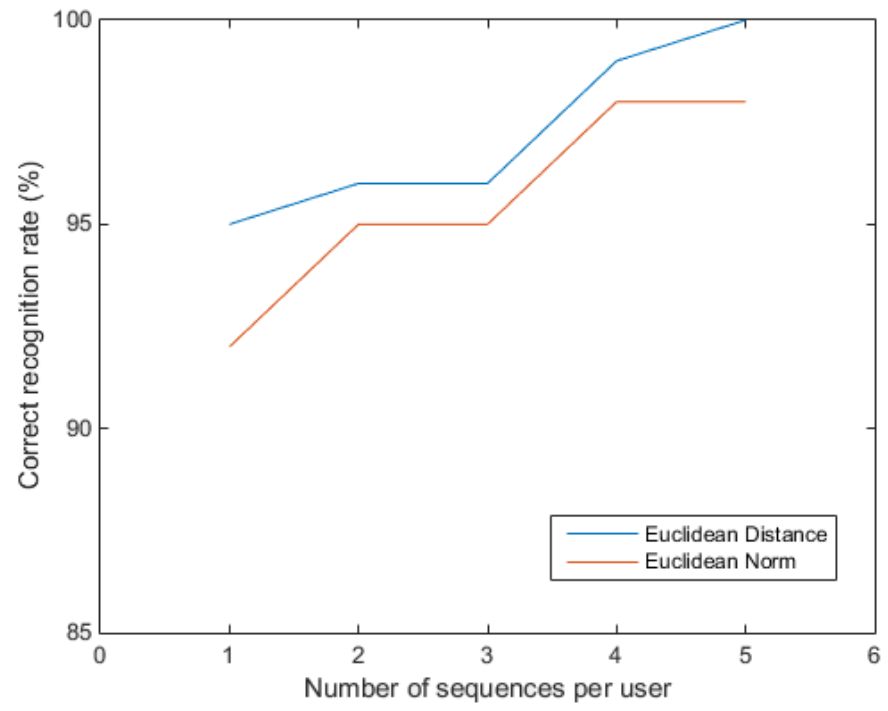

Fig. 6. Probe SEGI representation and the corresponding intensity values obtained with respect to a wrong user.

The original RPCA method has a high computational complexity. However, in the proposed method, a variation of the RPCA presented in [29] is used. The method is computationally inexpensive and takes $0.03 \mathrm{~s}$ to generate the SEGI representations for a user when using a context of 4 GEIs. The computations are performed on an Intel core i7 CPU (a) 3.60GHz. The average time to perform user recognition using the CASIA database using the Euclidean norm approach is $9.5 \mathrm{~s}$, while using it takes $9.6 \mathrm{~s}$ when the Euclidean distance approach is used.

The Multiscale Method proposed in [18] applies Gaussian filtering to a GEI at different scales. It then selects 500 random principal components, using 2D PCA, followed by 2D LDA. The method is repeated 10 times selecting a new set of 500 random principal components at every iteration. The recognition is performed by a majoring voting policy. The process is called Weighted RSL and the average time to perform user recognition using the same database is reported by the authors to be $52.8 \mathrm{~s}$, on Intel core i7 CPU@2.93 GHz. However, the average time required just by the WRSL recognition module, when implemented on Intel core $17 \mathrm{CPU}$ (a) $3.60 \mathrm{GHz}$ using GEIs as features is $20.8 \mathrm{~s}$, more than double of the proposed feature extraction and recognition based on SEGI.

\section{CONCLUSION}

The paper presents a new gait representation called SEGI to perform gait recognition. The SEGI representations for the gallery database are obtained by applying RPCA to the GEIs belonging to a single user. Given a probe GEI, the SEGI representation of the probe is first generated with respect to each user. Recognition can then be performed by computing the Euclidean norm of the probe SEGIs or by computing the Euclidean distance between the probe SEGI and the gallery SEGIs with respect to each user.

From the results, it can be concluded that the proposed approaches to use SEGI for recognition are equivalent to the state-of-the-art in terms of recognition performance, even when 
relying on very simple recognition methods. The simplicity of the recognition module highlights the effectiveness of the proposed SEGI representation as a gait feature. In future work, more powerful recognition modules will be considered in conjunction with the SEGI representation.

Although the proposed gait representation is effective for normal sequences, its performance under appearance change and change in view is yet to be tested. Appearance changes are caused by wearing different clothes or carrying items that are not available in the gallery, while view changes because the features made available to the camera to appear different from the ones available in the gallery. It is expected that these changes will be easily detected in a SEGI representation. Thus, the future work will include exploring SEGI in such unconstrained environments and testing on other available databases.

\section{REFERENCES}

[1] D. Gafurov, "A survey of biometric gait recognition: Approaches, security and challenges," in Annual Norwegian computer science conference, pp. 19-21, 2007.

[2] R. Sohrab, and B. Mahdi, "Human gait recognition using body measures and joint angles," international journal of scientific knowledge computing and information technology, 4th ed., vol. 6 , p. 2305,2015 .

[3] W. Yufei, S. Jiande, L. Jing and Z. Dong, "Gait recognition based on 3D skeleton joints captured by kinect," in Image Processing (ICIP), 2016 IEEE International Conference on, 2016.

[4] G. Zhao, G. Liu, H. Li, and M. Pietikainen, "3D gait recognition using multiple cameras," in 7th International Conference on Automatic Face and Gesture Recognition (FGR06), 2006.

[5] Y. Iwashita, R. Kurazume, R. Baba and K. Ogawara, "Method for gait-based biometric identification robust to changes in observation angle," in 26th International Conference of Image and Vision Computing, New Zealand, Auckland, 2011

[6] R. Bodor, A. Drenner, D. Fehr, O. Masoud, and N Papanikolopoulos, "View-independent human motion classification using image-based reconstruction," Image and Vision Computing, $8^{\text {th }}$ ed., vol. 27, , pp. 1194-1206, 2009.

[7] M. Goffredo, I. Bouchrika, J. Carter and M. Nixon, "Selfcalibrating view-invariant gait biometrics," IEEE Transactions on Systems, Man, and Cybernetics, $4^{\text {th }}$ ed., vol. 40, pp. $997-1008$, 2010.

[8] F. Jeana, A. B. Albub and R. Bergevina, "Towards view-invariant gait modeling:computing view-normalized body," Pattern Recognition, $11^{\text {th }}$ ed., vol. 42, pp. 2936-2949, 2009.

[9] M. Ju and B. Bir, "Individual recognition using gait energy image," IEEE transactions on pattern analysis and machine intelligence, $2^{\text {nd }}$ ed., vol. 28, pp. 316-322, 2006.

[10] J. Fazenda, D. Santos, and P. Correia, "Using gait to recognize people," in The International Conference on Computer as a Tool, EUROCON, Belgrade, 2005.

[11] K. Uchino, R. Kurazume, and Y. Iwashita, "Gait-based person identification robust to changes in appearance," Sensors - Open Access Journal, $6^{\text {th }}$ ed., vol. 13, pp. 7884-7901, 2013.

[12] W. Kusakunniran, Q. Wu, H. Li, and J. Zhang, "Multiple views gait recognition using view transformation model based on optimized gait energy image," in 12th International Conference on Computer Vision Workshops (ICCV Workshops), 2009 IEEE, pp.1058-1064, 2009.
[13] W. Kusakunniran, Q. Wu, H. Li, and J. Zhang, "Support vector regression for multi-view gait recognition based on local motion feature selection," in Computer Vision and Pattern Recognition (CVPR), 2010 IEEE Conference on, 2010.

[14] W. Kusakunniran, Q. Wu, H. Li, and J. Zhang, "Cross-view and multi-view gait recognitions based on view transformation model using multi-layer perceptron," Pattern Recognition Letters, $7^{\text {th }}$ ed., vol. 33, pp. 882-889, 2012.

[15] N. Liu, J. Lu and Y. P. Tan, " Joint subspace learning for viewinvariant gait recognition," IEEE Signal Processing Letters, $18^{\text {th }}$ ed., vol. 7, pp. 431-434, 2011.

[16] H. Chaubey, M. Hanmandlu and S. Vasikarla, "Enhanced view invariant gait recognition using feature level fusion," in Applied Imagery Pattern Recognition Workshop (AIPR), Washington, DC, 2014.

[17] Y. Guan, C.-T. Li and Y. Hu, "An adaptive system for gait recognition in multi-view environments," in 14th ACM Multimedia and Security Workshop, Coventry, UK, 2012.

[18] D. Choudhury, Sruti and T. Tardi, "Robust view invariant multiscale gait recognition," Pattern Recognition, $3^{\text {rd }}$ ed., vol. 48 , pp. 798-811, 2015.

[19] B. Khalid, X. Tao and G. Shaogang, "Cross View Gait Recognition using Correlation strength," in British Machine Vision Conference, London, 2010.

[20] T. T. Verlekar, P. Correia and L. D. Soares, "View-invariant gait recognition exploiting spatio-temporal information and a dissimilarity metric," in International Conf. of the Biometrics Special Interest Group - BIOSIG, Darmstadt, 2016.

[21] T. Connie, M.K. Goh, A.B. Teoh, "A grassmannian approach to address view change problem in gait recognition," IEEE Transactions on Cybernetics, 2016.

[22] K. Bashir, X. Tao and G. Shaogang, "Gait recognition using gait entropy image," in 3rd International Conference on Crime Detection and Prevention (ICDP ), 2009.

[23] N. Jain, M. Jeevan, M. Hanmandlu and G. Chetty, "Gait recognition based on gait pal and pal entropy image," in 20th IEEE International Conference on Image Processing (ICIP), IEEE, 2013.

[24] Y. Pratheepan, J. V. Condell and G. Prasad, "P rw gei: Poisson random walk based gait recognition," in IEEE 7th International Symposium on Image and Signal Processing and Analysis (ISPA), 2011

[25] Z. Lv, X. Xing, K. Wang, and D. Guan, "Class energy image analysis for video sensor-based gait recognition: a review," Sensors, vol. 15, pp. 932-964, 2015.

[26] E. Candès,X. Li, Y. Ma, and J. Wright, "Robust principal component analysis?," Journal of the ACM (JACM), $3^{\text {rd }}$ ed., vol. 58 , p. $11,2011$.

[27] X. Luan, B. Fang, L. Liu, W. Yang, J. Qian, "Extracting sparse error of robust PCA for face recognition in the presence of varying illumination and occlusion," Pattern Recognition, $2^{\text {nd }}$ ed., vol. 47, pp. 495-508, 2014.

[28] X. Luan, and W. Li, "Facial feature extraction based on robust pca and histogram," in Chinese Conference on Pattern Recognition, 2014.

[29] P. Rodriguez, and B. Wohlberg, "Fast principal component pursuit via alternating minimization," in 2013 IEEE International Conference on Image Processing, pp. 69-73, 2013.

[30] "CASIA Gait Database," http://www.sinobiometrics.com. 